\title{
Maternal heavy alcohol use and toddler behavior problems: a fixed effects regression analysis
}

\author{
Ann Kristin Knudsen • Eivind Ystrom • \\ Jens Christoffer Skogen $\cdot$ Leila Torgersen
}

Received: 10 October 2014 / Accepted: 6 January 2015 / Published online: 14 January 2015

(C) The Author(s) 2015. This article is published with open access at Springerlink.com

\begin{abstract}
Using data from the longitudinal Norwegian Mother and Child Cohort Study, the aims of the current study were to examine associations between postnatal maternal heavy alcohol use and toddler behavior problems, taking both observed and unobserved confounding factors into account by employing fixed effects regression models. Postnatal maternal heavy alcohol use (defined as drinking alcohol 4 or more times a week, or drinking 7 units or more per alcohol use episode) and toddler internalizing and externalizing behavior problems were assessed when the toddlers were aged 18 and 36 months. Maternal psychopathology, civil status and negative life events last year were included as time-variant covariates. Maternal heavy alcohol use was associated with toddler internalizing and externalizing behavior problems $(p<0.001)$ in the population when examined with generalized estimating equation models. The associations disappeared when observed and unobserved sources of confounding were taken into account in the fixed effects models [ $(p=0.909$ for externalizing behaviors $(b=0.002, \mathrm{SE}=0.021), p=0.928$ for internalizing
\end{abstract}

A. K. Knudsen $(\varangle)$

Department of Global Public Health and Primary Care, University of Bergen, Kalfarveien 31, 5018 Bergen, Norway e-mail: Ann.Knudsen@igs.uib.no

\section{A. K. Knudsen}

Department of Health Registries, Norwegian Institute of Public

Health, Bergen, Norway

\section{E. Ystrom}

Department of Genetics, Environment and Mental Health,

Norwegian Institute of Public Health, Oslo, Norway

E. Ystrom

Department of Psychology, Faculty of Social Sciences,

University of Oslo, Oslo, Norway behaviors $(b=0.002, \mathrm{SE}=0.023)$ ], with an even further reduction of the estimates with the inclusion of time-variant confounders. No causal effect was found between postnatal maternal heavy alcohol use and toddler behavior problems. Increased levels of behavior problems among toddlers of heavy drinking mothers should therefore be attributed to other adverse characteristics associated with these mothers, toddlers and families. This should be taken into account when interventions aimed at at-risk families identified by maternal heavy alcohol use are planned and conducted.

Keywords Heavy alcohol use - Fixed effects models . Repeated measures $\cdot$ Behavior problems

\section{Background}

One of the earliest developmental tasks is the evolvement of appropriate emotional and behavioral regulation in the toddler years [1]. The quality of the toddler's maternal

\author{
J. C. Skogen \\ Department of Public Mental Health, Norwegian Institute \\ of Public Health, Bergen, Norway \\ J. C. Skogen \\ Drug Research Western Norway, Stavanger University Hospital, \\ Stavanger, Norway \\ L. Torgersen \\ Department of Psychosomatics and Health Behavior, Norwegian \\ Institute of Public Health, Oslo, Norway
}


relationship and the social context under which the child grows up, are of extraordinary importance for optimal child development [2], but may be disturbed by maternal dysfunctional behavior [3]. One potentially important risk factor for maternal dysfunction in the toddler years is heavy alcohol use. Several studies have reported associations between heavy maternal alcohol use and increased levels of internalizing and externalizing behavior problems and lower social competence among school-age children [4-6], and adolescents and adults [7, 8]. Maternal risky alcohol use before [9] and during [10] pregnancy has also been linked to early development of behavior problems. However, in studies on toddlers and preschool children, the focus has primarily been on the impact of the father's heavy alcohol use on toddler behavior problems [11-15]. Studies that have included the role of maternal heavy alcohol use have examined this in addition to heavy paternal alcohol use [11-13, 15], with equivocal findings in regard to whether maternal heavy alcohol use further increases the risk of adverse child outcomes. The independent role of maternal heavy alcohol use on behavior problems in the toddler years is thus largely unknown, and is therefore the focus of the present study.

Children who grow up in families where either one or both of the parents are heavy alcohol users are also exposed to a range of other factors that may have adverse impact on their function and development. Although associations between parental heavy alcohol use and child and adolescents behavioral problems have been reported in several studies, the extent to which these associations reflect causal linkages is not known. There are two different processes that could result in an observed association between maternal heavy alcohol use and toddler behavior problems. First, the association could reflect a cause and effect linkage. Alcohol affects emotion regulation, cognitive functioning, judgement and impulse control; factors that all are important when taking care of and raising children. By influencing these factors, heavy maternal alcohol use may have a direct impact on parenting behavior, for instance by reducing the ability of a timely, responsive and stable parenting of the child [16], which again may affect the child's risk of developing behavior problems. Heavy maternal alcohol use may affect attachment quality [10] and reduce the mother's awareness, attention and sensitivity towards her child [17]. Children of heavy drinking mothers may also experience inconsistent disciplining [17], and they are at increased risk of being exposed to neglect and abuse [18]. Thus, through the mediating effect of parenting behavior, heavy maternal alcohol use may have a causal effect on toddler behavior problems. One could also imagine situations where heavy maternal alcohol use has a direct impact on toddler behavior problems. Being exposed to an intoxicated mother may cause feelings of confusion, insecurity and fear in the child, for instance in situations where the intoxication has lead to child abuse or neglect [18]. In a sample of children whose fathers were in treatment for alcoholism, Andreas and O'Farrell [19] found that the level of child maladjustment varied according to the father's current alcohol use status. The results from this study may indicate a causal role, in which parental heavy alcohol use acutely affect child behavior problems.

An alternative explanation is that the association between maternal heavy alcohol use and child behavior problems is non-causal, and rather reflects the presence of important confounders. A myriad of heritable [20], contextual [21], emotional [22] and behavioral factors associated with both heavy alcohol use and toddler behavior problems may be present in these mother-child constellations. Among the most acknowledged confounders in the association between maternal alcohol use and offspring outcomes are negative affectivity and maternal psychopathology [such as anxiety, depression and attention deficit hyperactivity disorder (ADHD)], outgoing personality, marital conflict, presence of a heavy drinking partner, and a generally more disorganized and chaotic lifestyle [11, 23-28]. Prenatal alcohol use is a particularly important factor associated with both postnatal alcohol use and child behavior problems [10]. Studies that have examined the effect of postnatal maternal alcohol use on offspring outcomes have been limited in their ability to control for this important confounder. Finally, alcohol use disorders includes a genetic component, and women engaging in risk drinking may also transfer genetic vulnerability to their children, which may be expressed as behavior problems in the child [20, 29]. It is extremely difficult to define and measure all these potential confounders exhaustively in a single epidemiological study [30]. As previous studies have had scarce access to data beyond exposure and outcome variables, they have not been able to separate a potential causal effect of maternal heavy alcohol use from confounding effects.

In the present study, we applied fixed effects models as a method that can mitigate the challenges of unmeasured confounders. In repeated measured data, fixed effects models can eliminate between-individual differences (such as genetic, contextual, functional or personality differences between mothers) and show the effect of within-individual changes (i.e., whether a change in heavy alcohol use in mother A is associated with changes in behavioral problems in toddler A). Sources of variations in exposures and outcomes present before and at the first measurement, and for which the effect is likely to be stable over time ("timeinvariant factors"), are in this model considered as fixed at the first measurement, and can therefore be controlled for using the individual person as its own control [30]. Measuring of these potential confounders is therefore unnecessary. Fixed effects regression models thus estimate the 
within-individual exposure-outcome association, taking both observed and unobserved confounders that are specific to the individual participant into account [31]. However, heavy alcohol use is also associated with a disorganized and chaotic lifestyle, relationship instability and psychopathology [28]. Factors such as changes in maternal civil status, mental health, or recent experience of negative life events may also change between the measures, and affect both maternal heavy alcohol use status and toddler behavior problems. Such time-variant variables may also be controlled for in fixed effects regression models, and these models thus allows for the control of both time-invariant confounders and observed time-variant covariates.

By employing fixed effect regression models on a national representative sample of 51,000 children, followed from birth until age 36 months, we aimed in the present study to establish whether maternal heavy alcohol use and toddler behavioral problems are independently associated. This is an important step in ascertaining a potential causal linkage between maternal alcohol use and toddler behavior problem. More specifically, we wanted to examine: (1) whether an association between maternal heavy alcohol use and behavioral problems in the toddler years can be found in the general population, (2) whether an association between maternal heavy alcohol use and toddler behavioral problems are partly or fully explained by observed and unobserved sources of confounding, and (3) whether an association is further attenuated by taking the potential time-variant confounders of maternal mental distress, changes in civil status and experience of negative life events into account.

\section{Materials and methods}

\section{Study design and participants}

The data examined in the present study were derived from the Norwegian Mother and Child Cohort Study (MoBa). $\mathrm{MoBa}$ is a prospective population-based pregnancy cohort study conducted by the Norwegian Institute of Public Health [32]. Participants were recruited from all over Norway from 1999 to 2008, and women consented to participation in $40.6 \%$ of the invited pregnancies. The cohort now includes 114,500 children, 95,200 mothers and 75,200 fathers.

The primary measures were conducted at gestation week 17/18, with follow-up by questionnaires at regular intervals. The current study is based on version seven of the quality-assured data files released for research in 2013. The current study has a repeated measures design, and employs information reported by the mothers when the child was 18 months (Questionnaire 5, Q5), and 36 months old (Questionnaire 6, Q6). The main inclusion criterion was valid information on the outcome measures in both questionnaires, fulfilled by $N=51,891$ of the mothers. The second and third child in twin or triplet births $(n=770)$, and children with missing information on gender $(n=6)$ were excluded from the sample. The total population eligible for the current study was $N=51,115,46.8 \%$ of those who participated at least once in the MoBa study.

\section{Maternal heavy alcohol use}

Mothers who either drank alcohol frequently, or who drank excessive quantities of alcohol when they were drinking, assessed at child age 18 and 36 months, were defined as heavy alcohol users. Frequency was measured with the item "How often do you consume alcohol?" with alternatives ranging from "never" to "roughly 6-7 times a week", while quantity were assessed for both weekend and weekdays through the item "How many units do you usually drink when you consume alcohol?" with fixed answer categories ranging from "less than 1", to "10 or more". In MoBa, one unit was defined as equivalent to $1.5 \mathrm{cl}$ (12.8 g) of pure alcohol.

Although not a fully valid measure of alcohol problems per se [33], frequency of alcohol use may be an indicator of alcohol abuse and dependence [34]. Among Norwegian women, alcohol use is most common in the weekend evenings [35]. Weekday alcohol use has been found to be more strongly associated with alcohol problems than weekend alcohol use [36], probably because those who engage in weekday alcohol use are more likely to be heavy drinkers [36]. We therefore categorized those who reported to be drinking alcohol four times a week or more as heavy drinkers in terms of frequency, as these mothers were likely to drink both during weekends and weekdays.

Risky single-occasion alcohol use among women is commonly referred to as the intake of approximately 40-60 g pure alcohol ( $\approx 3.1-4.7$ in MoBa alcohol units) or more in a single occasion [34, 37]. However, the level of intoxication depend on factors such as duration of the drinking occasion, age, body weight and whether or not the alcohol is consumed together with food [37]. In the present context, we aimed to identify mothers truly at risk for intoxication, and we thus categorized mothers who reported that they usually drank seven or more alcohol units as "heavy drinkers".

Heavy alcohol use in the present context was thus defined as (1) alcohol use four or more times a week, or (2) usually consumption of seven alcohol units or more when drinking. Approximately, $1 \%$ of the mothers answered affirmatively on the first category, while $3 \%$ of the mothers reported the second category.

Toddler behavior problems

Toddler behavior problems were assessed in the 18and 36-month questionnaires with items from the Child 
Behavior Checklist version for preschool children (CBCL/1.5-5) [38]. CBCL/1.5-5 is constructed to cover a range of emotional, social and behavior problems, and consists of 99 items describing child behavior in the preceding 2 months. CBCL/1.5-5 has several subscales, which again are included in two scales measuring internalizing and externalizing behavior problems. The CBCL version for older children has been validated in a Norwegian study [39], while the CBCL/1.5-5 has been validated in Dutch and Danish samples [40, 41].

In common with most large inter-disciplinary epidemiological studies, item selection was necessary due to space restriction in the MoBa questionnaires. The selected CBCL items represented subscales of the internalizing domain ("emotionally reactive," "anxious/depressed," and "somatic complaints") and externalizing domain ("attention problems" and "aggressive behavior"). The item selection procedure was based on consensus among specialists in clinical and developmental psychology and aimed at representing each subscale with items that were both clinically and theoretically relevant. Thirteen of the items were included in both questionnaires, and were used in the current study as indicators of internalizing (five items) and externalizing behavior problems (eight items). The mothers were asked to rate each item on a scale from 0 "not true", 1 "somewhat or sometimes true" to 2 "very true or often true". Sum scores were calculated for each subscale. In cases were responses were missing for one or two items, sum scores were calculated based on the mean score on the items that were responded to. Scores for internalizing problems ranged from 0 to 10 , while scores for externalizing problems range from 0 to 16 . To assess changes in total scale scores that were not due to normative changes between 18 and 36 months, the scores in the internalizing and externalizing problem variables were standardized for each age group.

Time-variant covariates

A selection of variables that appeared in the time-period between the 18- and 36-month assessments, and which were considered likely to affect both maternal heavy alcohol use and toddler behavior problems were included as potential time-variant confounders. These are presented below.

\section{Maternal civil status}

The mother was asked to report her current civil status. Based on her response, a dichotomous variable was conducted, indicating whether the mother was currently (0) in a relationship or (1) single.

\section{Maternal mental distress}

Maternal mental distress was assessed with the eight items version of Hopkins Symptom Check-List (SCL-8) [42]. For each item, the respondents were asked to indicate how much any of the presented emotions and cognitions had bothered them during the past 2 weeks, scored on a fourpoint scale ranging from 1 "not bothered" to 4 "very bothered". The item scores were then summarized into a continuous variable, ranging from 8 to 32 . For participants with missing responses on one, two or three items, the sum score was based on the summarized mean value of the items responded to.

\section{Negative life events}

Four variables on negative life events last year were included (yes/no): (1) whether the mother herself had been seriously ill or injured, (2) whether anyone close to the mother had been seriously ill or injured, (3) whether the mother had been involved in a serious accident, fire or robbery, or (4) whether the mother had lost someone close to her.

\section{Statistical procedures}

Missing responses in the exposure variables and time-variant variables ranged from 0.3 (civil status at 18 months) to $5.3 \%$ (negative life events at 36 months). Multiple missing imputation procedures in Stata 12.0 [43], using the multivariate chained approach with ten imputation procedures, were employed before the main analyses were conducted.

The pooled population-averaged associations between maternal heavy alcohol use and age-standardized levels of internalizing and externalizing behaviors were investigated with generalized estimating equation (GEE) methods [44]. Next, we used fixed effects regression models to examine the within-individual associations between maternal heavy alcohol use and toddler behavior problems, controlled for observed and unobserved confounding factors. The use of a fixed effects model allows to estimate the effect of changes in maternal heavy alcohol use status from time 1 (18 months) to time 2 (36 months) on changes in toddlers level of behavioral problems, holding all the observed and unobserved sources of individual variation in heavy alcohol use status and toddler behavior problems at 18 months constant. Finally, we included the time-variant observed factors in the fully adjusted models. All associations were adjusted for pregnancy at the 18- or 36-month questionnaires. Estimates are presented as coefficients $(b)$, with standard errors (SE). Statistical significance is reported with $p$ values. All analyses were conducted in Stata 12.0 [45]. 
Table 1 Descriptive characteristics on fixed effects at toddler age 18 months, and exposure variables, outcome variables and time-variant covariates of the MoBa participants at toddler age 18 and 36 months

\begin{tabular}{|c|c|c|c|c|}
\hline & \multicolumn{2}{|l|}{18 months } & \multicolumn{2}{|l|}{36 months } \\
\hline & $n(\%)$ & Mean (SD) & $n(\%)$ & Mean (SD) \\
\hline \multicolumn{5}{|l|}{ Fixed characteristics } \\
\hline Child gender, boy ${ }^{\mathrm{a}}$ & $25,006(48.9)$ & & & \\
\hline Birth weight $(\mathrm{g})^{\mathrm{a}}$ & & $3,585.3(562.9)$ & & \\
\hline Gestation length (weeks) ${ }^{\mathrm{a}}$ & & $39.5(1.8)$ & & \\
\hline Maternal age at birth (years) ${ }^{\mathrm{a}}$ & & $30.4(4.4)$ & & \\
\hline Number of older siblings ${ }^{\mathrm{a}}$ & & $0.7(0.8)$ & & \\
\hline Completed education mother, high school or less ${ }^{\mathrm{a}}$ & $14,584(30.1)$ & & & \\
\hline Household income before pregnancy, $<400,000 \mathrm{NOK}$ & $10,416(21.7)$ & & & \\
\hline \multicolumn{5}{|l|}{ Alcohol consumption during pregnancy (yes) } \\
\hline Week $0-12$ & $13,306(26.5)$ & & & \\
\hline Week 13-24 & $4,444(10.0)$ & & & \\
\hline Week $25+$ & $5,034(10.0)$ & & & \\
\hline Paternal heavy alcohol use $e^{\mathrm{b}, \mathrm{c}}$ & $8,710(21.3)$ & & & \\
\hline \multicolumn{5}{|l|}{ Exposure } \\
\hline Maternal heavy alcohol use ${ }^{b}$ & $2,146(4.3)$ & & $2,237(4.5)$ & \\
\hline Missing & $694(1.4)$ & & $1,445(2.8)$ & \\
\hline \multicolumn{5}{|l|}{ Outcomes } \\
\hline Internalizing problems ${ }^{\mathrm{d}}$ & & $1.3(1.2)$ & & $1.4(1.4)$ \\
\hline Externalizing problems ${ }^{\mathrm{d}}$ & & $3.9(2.2)$ & & $3.9(2.4)$ \\
\hline \multicolumn{5}{|l|}{ Time-variant covariates } \\
\hline Civil status (single) & $1,645(3.2)$ & & $2,214(4.4)$ & \\
\hline Missing & $150(0.3)$ & & $1,019(2.0)$ & \\
\hline Maternal mental distress ${ }^{\mathrm{e}}$ & & $10.2(2.9)$ & & $10.2(3.1)$ \\
\hline Missing & $569(1.1)$ & & $1,040(2.0)$ & \\
\hline Negative life events last year (one or more) & & $12,672(25.3)$ & & $12,859(26.6)$ \\
\hline Missing & $1,098(2.2)$ & & $2,727(5.3)$ & \\
\hline
\end{tabular}

Characteristics only presented for participants with valid responses

Total sample size $n=51,115$

${ }^{a}$ When the child is born

b Heavy alcohol use defined as (a) drinking alcohol four or more times a week, or (b) drinking seven or more alcohol units when they are drinking

c 6 months before or during partner's pregnancy

d Sum score, not age standardized

e Score on SCL-8

Ethics

License to conduct the MoBa study was obtained from the Norwegian Data Inspectorate, and the current study was approved by the Regional Committee for Medical Research Ethics. Informed consent was obtained from all participants.

\section{Results}

The characteristics of the sample before multiple missing imputation procedures, including some of the fixed characteristics at 18 months are presented in Table 1 . When the toddler was aged 18 months, $4.3 \%$ of the mothers reported heavy alcohol use, compared to $4.5 \%$ at toddler age 36 months (Table 1). While $2 \%$ of the samples were heavy drinkers at both assessments, slightly more than 2,300 mothers (4.7 \%) changed heavy alcohol use status from 18 months to 36 months (data not shown). In the total sample, $2.2 \%$ of the mothers ceased heavy alcohol use behavior while $2.5 \%$ started using alcohol heavily from toddler age from 18 months to 36 months. Mean level of internalizing and externalizing behavior problems was similar at 18 and 36 months (Table 1). More mothers were 
Table 2 Associations between maternal heavy alcohol use and toddler externalizing and internalizing behaviors

\begin{tabular}{|c|c|c|c|c|}
\hline & \multicolumn{2}{|c|}{ Externalizing behaviors $^{\mathrm{c}}$} & \multicolumn{2}{|c|}{ Internalizing behaviors $^{\mathrm{c}}$} \\
\hline & $b(\mathrm{SE})$ & $p$ & $b(\mathrm{SE})$ & $P$ \\
\hline Pregnancy adjusted ${ }^{\mathrm{a}}$ & $0.171(0.015)$ & $<0.001$ & $0.111(0.015)$ & $<0.001$ \\
\hline Fixed effects model & $0.002(0.021)$ & 0.909 & $0.002(0.023)$ & 0.928 \\
\hline Adjusted for fixed effects and time-variant covariates ${ }^{b}$ & $-0.001(0.021)$ & 0.968 & $-0.003(0.023)$ & 0.894 \\
\hline
\end{tabular}

Regression analyses adjusted for pregnancy, fixed effects, and fixed effects and time-variant covariates

${ }^{a}$ Generalized estimating equation (GEE) model

b Adjusted for concurrent maternal civil status, concurrent maternal mental distress and concurrent maternal experience of negative life events last year

${ }^{c}$ Age standardized

single, had experienced a negative life event and had higher levels of mental distress when the toddler was 36 months compared to 18 months (Table 1).

The results from the analyses employing GEE and fixed effects regression models are presented in Table 2. The GEE models indicated a positive association in the population between maternal heavy alcohol use and toddler behavior problems $(p<0.001$ for both internalizing $(b=0.171, \mathrm{SE}=0.015)$ and externalizing behaviors $(b=0.111$, SE $=0.015)$. However, the associations disappeared when observed and non-observed sources of confounding were taken into account in the fixed effects models $[(p=0.909$ for externalizing behaviors $(b=0.002$, $\mathrm{SE}=0.021), p=0.928$ for internalizing behaviors $(b=0.002, \mathrm{SE}=0.023)$ ], with an even further reduction of the estimates when maternal civil status, maternal mental distress and experience of negative life events were included in the model.

\section{Discussion}

Based on a large birth cohort from the general population, we found that the average toddler of a heavy alcohol use mother had heightened levels of behavior problems. On the individual level, however, we found no indication of a causal effect between maternal heavy alcohol use and toddler behavior problems, as the increased levels of toddler behavior problems found in the population were completely explained by controlling for observed and unobserved confounders in a fixed effect model.

Although the need for knowledge about the effect of postnatal maternal heavy alcohol use on child outcomes has been emphasized [10], the literature in this area is scarce. Research that has examined the effect of parental heavy alcohol use on behavior problems in toddlers is in general limited, and the role of heavy alcohol use toddler mothers has, to the best of our knowledge, not been studied independently of paternal heavy alcohol use. Previous studies have indicated that toddlers and children whose both parents or fathers engage in heavy alcohol use have more behavior problems [4, 5, 11-15]. In the present study, we found higher levels of externalizing and internalizing behavior problems in the population among toddlers of mothers who engaged in heavy alcohol use. The increased problems were, however, completely explained by observed and unobserved sources of confounding. This finding is in line with studies examining the genetic versus the environmental risk for internalizing and externalizing behavior problems in adolescent children of alcohol abusing parents, using the children-of-twins (COT) design. A recent systematic review of these studies by McAdams and colleagues concluded that parental alcohol abuse had no significant environmental influence on offspring mental problems in adolescence [46]. Although toddlers in our sample of heavy alcohol use mothers are at increased risk, this risk seems to be related to other adverse characteristics of the mother, family or toddler and not to the heavy alcohol use per se.

Our findings indicates not only lack of effect of maternal heavy alcohol use on toddler behavior problems, but also a lack of effect from other factors that may temporarily or acutely be affected by heavy maternal alcohol use, such as parenting behavior, attention, patience, responsiveness or warmth toward the child. However, as fixed effects models are developed to examine the effect of within-individual changes (i.e., the effect of changes from low/moderate to heavy alcohol use), we cannot study the effect of stable heavy drinking in these models. Further, we did not have information on to what degree the toddlers had been exposed to the mothers heavy drinking. The maternal heavy alcohol use may have only taken place when the toddler was not present, for instance after bedtime or when the mother had a babysitter. A higher level of family functioning and positive parenting styles may also have been protective for the negative effect of heavy maternal alcohol use [47]. We also did not have information about the duration of the period in which the mother engaged in heavy alcohol use. We can thus not exclude the possibility that toddlers 
who are directly and over time exposed to a heavy drinking mother will show different behavior patterns than found in our study.

The increased level of behavior problems identified at the population level among toddlers of heavy alcohol use mothers could have practical implications. Maternal heavy alcohol use is a potential indicator for vulnerable families and toddlers at risk, even if the increased risk is completely explained by other adverse factors. Screening for alcohol problems is part of routine assessments for pregnant women, and could easily be included as part of the general postnatal assessment. Interventions for families with heavy alcohol use mothers should take a broad perspective, and be aimed at a range of potential adverse characteristic. Based on our results, it is unlikely that interventions aimed to only help the mother to cut-down her alcohol use will have an effect on the toddler's behavior problems.

\section{Strengths and limitations}

There are three important sources of bias in epidemiological research: confounding, measurement errors and selective participation. The main strength of the present study is the ability to control for all potential confounders, both observed and unobserved, at the 18-month assessment with the use of a repeated measures design analyzed with fixed effects models. This method made it possible for ascertaining a potential causal linkage between maternal alcohol use and toddler behavior problem. Another strength is the large sample size taken from a general population of toddler mothers, which allowed us to study the effect of heavy maternal alcohol use in the general population, and not only among clinical cases.

Despite that the fixed effects model allows for control of all confounding factors up to age 18 months that has effect on both time points, some factors may still operate as time-specific confounders at 18 and 36 months. We controlled for three such sources of confounders; namely changes in maternal civil status, changes in maternal mental distress and experience of negative life events between the 18- and 36-months assessments. There may also be other factors operating as residual confounders. One such factor is changes in paternal alcohol consumption, which may be associated with both heavy maternal drinking and behavior problems in the offspring [10, 12, 13, 25, 48]. Unfortunately, we did not have information on paternal alcohol consumption at toddler age 18 and 36 months, but the stable effect of paternal drinking was adjusted for in the fixed effects analyses. However, as no association was left after controlling for the fixed effects at 18 months, including changes in paternal alcohol consumption would probably not have any important impact on the estimates found.
With regard to the other two sources of bias, the present study has some limitations. Under-reporting of alcohol consumption is a challenge in health studies [49] and is particularly common in studies based on pregnancy samples [50]. Thus, some individuals may have been misclassified as non-heavy drinkers in the present study. Our strict definition of heavy alcohol use in the present study (four or more times a week, or more than seven alcohol units when drinking) was constructed to identify mothers reporting the highest levels of alcohol consumption. Despite this strict definition, no effect was found on our outcome measures. As only $4 \%$ of the participating mothers satisfied our criteria, an increased threshold of heavy alcohol use may be of little public health relevance.

Even when employing a repeated measurement design with a time interval of 18 months, some cases of maternal heavy alcohol use may only have been present in the interval between the measurement points, and therefore not identified at the time of measurements. There is, however, no reason to believe that these mothers differ systematically from the mothers with heavy alcohol use at the measurement time points.

Another source of measurement error may be the noninclusion of the full CBCL $1.5 / 5$ in the MoBa questionnaires. While the items indicating behavior problems have been used previous publications from the MoBa database $[9,51]$, the abbreviated versions of the original scales are not validated. We aimed to reduce this potential measurement bias by constructing age-standardized continuous scales of internalizing and externalizing behavior problems, and examine a dose-response relationship without need for a validated cutoff.

Selective participation is the third important source of bias in epidemiological studies. The results in the present study are based on data from an extensive longitudinal study with several repeated measures. Continued participation may be associated with a high level of general functioning, and heavy alcohol use mothers who participated in MoBa may therefore differ from nonparticipating heavy alcohol use mothers [52]. However, a study of self-selection in MoBa concluded that the survey was relatively representative for the total population of pregnant women in Norway [53], and that selective nonparticipation was more likely to affect prevalence estimates than exposure-outcome associations. Further, the use of fixed effects models, with its focus on individual changes and control for all background variables, reduces bias associated with selective participation. Selection bias may therefore be of less of concern in the present study.

Finally, some limitations are related to the use of fixed effects models. Fixed effects models take only information on within-individual differences into account, discarding all information on between-individuals differences. If 
predictor variables vary greatly across, but not within, individuals over time, the fixed effect estimates become quite imprecise. Fixed effects models often produce estimates with large standard errors, leading to higher $p$ values and wider confidence intervals [54]. About 2,300 (4.7 \%) mothers changed their heavy alcohol use behavior from child age 18 to 36 months in the present study, and this should be a large enough sample to detect any effect of changes in alcohol use on toddler behavior problems. With standard errors in the fully adjusted fixed effects models at 0.021 for externalizing and 0.023 for internalizing behaviors, the true causal effects of maternal heavy alcohol use would have to be less than approximately 0.04 to not reach a $p$ value of $<0.05$. This would represent effects of little clinical or public health importance.

\section{Conclusion}

Increased levels of behavior problems among toddlers of mothers engaging in heavy alcohol use are probably not caused by the heavy alcohol use behavior per se, but should rather be attributed to a range of challenges and problematic characteristics associated with these mothers, toddlers and families. This should be taken into account when interventions aimed at at-risk families identified by maternal heavy alcohol use are planned and conducted.

Acknowledgments The Norwegian Mother and Child Cohort Study is supported by the Norwegian Ministry of Health, NIH/NIEHS (Grant No. N01-ES-85,433), NIH/NINDS (Grant No.1 UO1 NS 047,537-01) and the Norwegian Research Council/FUGE (Grant No. $151,918 / \mathrm{S} 10)$. We are grateful to all the participating families in Norway who take part in this on-going cohort study. No specific funding was given for this study.

\section{Conflict of interest None.}

Open Access This article is distributed under the terms of the Creative Commons Attribution License which permits any use, distribution, and reproduction in any medium, provided the original author(s) and the source are credited.

\section{References}

1. Zahn-Waxler C, Klimes-Dougan B, Slattery MJ (2000) Internalizing problems of childhood and adolescence: prospects, pitfalls, and progress in understanding the development of anxiety and depression. Dev Psychopathol 12:443-466

2. Lyons-Ruth K, Zeanah CH, Benoit D (2003) Disorder and risk for disorder during infancy and toddlerhood. In: Mash EJ, A. BR (eds) Child Psychopathology. The Guilford Press, New York

3. Schechter DS, Willheim E (2009) Disturbances of attachment and parental psychopathology in early childhood. Child Adol Psych Cl 18:665-686

4. Hussong AM, Flora DB, Curran PJ, Chassin LA, Zucker RA (2008) Defining risk heterogeneity for internalizing symptoms among children of alcoholic parents. Dev Psychopathol 20:165-193

5. Hussong AM, Wirth RJ, Edwards MC, Curran PJ, Chassin LA, Zucker RA (2007) Externalizing symptoms among children of alcoholic parents: entry points for an antisocial pathway to alcoholism. J Abnorm Psychol 116:529-542

6. Christensen HB, Bilenberg N (2000) Behavioural and emotional problems in children of alcoholic mothers and fathers. Eur Child Adolesc Psychiatry 9:219-226

7. Rognmo K, Torvik FA, Ask H, Røysamb E, Tambs K (2012) Paternal and maternal alcohol abuse and offspring mental distress in the general population: the Nord-Trøndelag Health Study. BMC Pub Health 12:448

8. Malone SM, McGue M, Iacono WG (2010) Mothers' maximum drinks ever consumed in 24 hours predicts mental health problems in adolescent offspring. J Child Psychol Psychiatry 51:1067-1075

9. Knudsen AK, Skogen JC, Ystrom E, Sivertsen B, Tell GS, Torgersen L (2014) Maternal pre-pregnancy risk drinking and toddler behavior problems: the Norwegian Mother and Child Cohort Study. Eur Child Adolesc Psychiatry 23:901-911

10. Olson HC, O’Connor MJ, Fitzgerald HE (2001) Lessons learned from study of the developmental impact of parental alcohol use. Inf Ment Hlth J 22:271-290

11. Keller PS, Cummings EM, Davies PT, Mitchell PM (2008) Longitudinal relations between parental drinking problems, family functioning, and child adjustment. Dev Psychopathol 20:195-212

12. Edwards EP, Eiden RD, Colder C, Leonard KE (2006) The development of aggression in 18 to 48 month old children of alcoholic parents. J Abnorm Child Psychiatry 34:409-423

13. Edwards EP, Leonard KE, Das Eiden R (2001) Temperament and behavioral problems among infants in alcoholic families. Inf Ment Health J 22:374-392

14. Edwards EP, Das Eiden R, Leonard KE (2006) Behavior problems in 18-to 36-month-old children of alcoholic fathers: secure mother-infant attachment as a protective factor. Dev Psychopathol 18:395-407

15. Eiden RD, Edwards EP, Leonard KE (2007) A conceptual model for the development of externalizing behavior problems among kindergarten children of alcoholic families: role of parenting and children's self-regulation. Dev Psychol 43:1187-1201

16. Mayes LC, Truman SD (2005) Substance abuse and parenting. In: Bornstein MH (ed) Handbook of parenting: Social conditions and applied parenting, vol 4. Lawrence Erlbaum Associates, New Jersey

17. Molina BSG, Donovan JE, Belendiuk KA (2010) Familial loading for alcoholism and offspring behavior: mediating and moderating influences. Alcohol Clin Exp Res 34:1972-1984

18. Gmel G, Rehm J (2003) Harmful alcohol use. Alcohol Res Health 27:52-62

19. Andreas JB, O'Farrell TJ (2007) Longitudinal associations between fathers' heavy drinking patterns and children's psychosocial adjustment. J Abnor Child Psychol 35:1-16

20. Knopik VS, Heath AC, Jacob T, Slutske WS, Bucholz KK, Madden PAF, Waldron M, Martin NG (2006) Maternal alcohol use disorder and offspring ADHD: disentangling genetic and environmental effects using a children-of-twins design. Psychol Med 36:1461-1471

21. Bijttebier P, Goethals E, Ansoms S (2006) Parental drinking as a risk factor for children's maladjustment: the mediating role of family environment. Psychol Add Behav 20:126-130

22. Preuss UW, Schuckit MA, Smith TL, Barnow S, Danko GP (2002) Mood and anxiety symptoms among 140 children from alcoholic and control families. Drug Alcohol Depend 67:235-242

23. Rehm J, Mathers C, Popova S, Thavorncharoensap M, Teerawattananon Y, Patra J (2009) Alcohol and global health 1 Global burden of disease and injury and economic cost attributable to alcohol use and alcohol-use disorders. Lancet 373:2223-2233 
24. Hicks M, Tough SC, Johnston D, Siever J, Clarke M, Sauve R, Brant R, Lyon AW (2014) T-ACE and predictors of self-reported alcohol use during pregnancy in a large, population based urban cohort. Int J Alcohol Drug Res 3:51-61

25. Torvik FA, Roysamb E, Gustavson K, Idstad M, Tambs K (2013) Discordant and concordant alcohol use in spouses as predictors of marital dissolution in the general population: results from the hunt study. Alcohol Clin Exp Res 37:877-884

26. Ystrom E, Vollrath ME, Nordeng H (2012) Effects of personality on use of medications, alcohol, and cigarettes during pregnancy. Eur J Clin Pharmacol 68:845-851

27. Stene-Larsen K, Torgersen L, Strandberg-Larsen K, Normann PT, Vollrath ME (2013) Impact of maternal negative affectivity on light alcohol use and binge drinking during pregnancy. Acta Obstet Gyn Scan 92:1388-1394

28. Hasin DS, Stinson FS, Ogburn E, Grant BF (2007) Prevalence, correlates, disability, and comorbidity of DSM-IV alcohol abuse and dependence in the United States-results from the National Epidemiologic Survey on Alcohol and Related Conditions. Arch Gen Psychiat 64:830-842

29. Kendler KS, Aggen SH, Knudsen GP, Roysamb E, Neale MC, Reichborn-Kjennerud T (2011) The structure of genetic and environmental risk factors for syndromal and subsyndromal common DSM-IV axis I and all axis II disorders. Am J Psychiatry 168:29-39

30. Gunasekara FI, Richardson K, Carter K, Blakely T (2014) Fixed effects analysis of repeated measures data. Int $\mathrm{J}$ Epidemiol 43:264-269

31. Fitzmauric GM, Laird NM, Ware JH (2011). Fixed effects versus random effects models. In: Applied longitudinal analysis. Wiley, New Jersey

32. Magnus P, Irgens LM, Haug K, Nystad W, Skjaerven R, Stoltenberg C (2006) Cohort profile: the Norwegian Mother and Child Cohort Study (MoBa). Int J Epidemiol 35:1146-1150

33. Gmel G, Heeb JL, Rehm J (2001) Is frequency of drinking an indicator of problem drinking? A psychometric analysis of a modified version of the alcohol use disorders identification test in Switzerland. Drug Alcohol Depend 64:151-163

34. Dawson DA (2011) Defining risk drinking. Alcohol Res Health 34:144-156

35. Sieri S, Agudo A, Kesse E, Klipstein-Grobusch K, San-Jose B, Welch AA, Krogh V, Luben R, Allen N, Overvad K, Tjonneland A, Clavel-Chapelon F, Thiebaut A, Miller AB, Boeing H, Kolyva M, Saieva C, Celentano E, Ocke MC, Peeters PHM, Brustad M, Kumle M, Dorronsoro M, Feito AF, Mattisson I, Weinehall L, Riboli E, Slimani N (2002) Patterns of alcohol consumption in 10 European countries participating in the European Prospective Investigation into Cancer and Nutrition (EPIC) project. Public Health Nutr 5:1287-1296

36. Room R, Makela P, Benegal V, Greenfield TK, Hettige S, Tumwesigye NM, Wilsnack R (2012) Times to drink: cross-cultural variations in drinking in the rhythm of the week. Int J Public Health 57:107-117

37. Gmel G, Kuntsche E, Rehm J (2011) Risky single-occasion drinking: bingeing is not bingeing. Addiction 106:1037-1045

38. Achenbach TM (1992) Manual for the child behavior checklist/2-3 and 1992 profile. University of Vermont, Burlington
39. Nøvik TS (1999) Validity of the child behaviour checklist in a Norwegian sample. Eur Child Adoles Psychiatry 8:247-254

40. Koot HM, VanDenOord E, Verhulst FC, Boomsma DI (1997) Behavioral and emotional problems in young preschoolers: crosscultural testing of the validity of the child behavior checklist/2-3. J Abnorm Child Psychol 25:183-196

41. Kristensen S, Henriksen TB, Bilenberg N (2010) The child behavior checklist for Ages 1.5-5 (CBCL/11/2-5): assessment and analysis of parent- and caregiver-reported problems in a population-based sample of Danish preschool children. Nord J Psychiat 64:203-209

42. Strand BH, Dalgard OS, Tambs K, Rognerud M (2003) Measuring the mental health status of the Norwegian population: a comparison of the instruments SCL-25, SCL-10, SCL-5 and MHI-5 (SF-36). Nord J Psychiat 57:113-118

43. McKnight PE, McKnight KM, Sidani S, Figueredo AJ (2007). Multiple imputation. In: Missing data. The Guildford, New York

44. Zeger SL, Liang KY, Albert PS (1988) Models for longitudinal data-a generalized estimating equation approach. Biometrics 44:1049-1060

45. StataCorp (2011). Stata 12 Base reference manual. Stata press

46. McAdams TA, Neiderhiser JM, Rijsdijk FV, Narusyte J, Lichtenstein P, Eley TC (2014) Accounting for genetic and environmental confounds in associations between parent and child characteristics: a systematic review of children-of-twins studies. Psychol Bull 140:1138-1173

47. Coyle JP, Nochajski T, Maguin E, Safyer A, DeWit D, Macdonald S (2009) An exploratory study of the nature of family resilience in families affected by parental alcohol abuse. J Fam Issues 30:1606-1623

48. El-Sheikh M, Flanagan E (2001) Parental problem drinking and children's adjustment: family conflict and parental depression as mediators and moderators of risk. J Abnorm Child Psychol 29:417-432

49. Stockwell T, Donath S, Cooper-Stanbury M, Chikritzhs T, Catalano P, Mateo C (2004) Under-reporting of alcohol consumption in household surveys: a comparison of quantity-frequency, graduated-frequency and recent recall. Addiction 99:1024-1033

50. Alvik A, Haldorsen T, Groholt B, Lindemann R (2006) Alcohol consumption before and during pregnancy comparing concurrent and retrospective reports. Alcohol Clin Exp Res 30:510-515

51. Stene-Larsen K, Borge AIH, Vollrath ME (2009) Maternal smoking in pregnancy and externalizing behavior in 18-month-old children: results from a population-based prospective study. J Am Acad Child Adolesc Psychiat 48:283-289

52. Knudsen AK, Hotopf M, Skogen JC, Overland S, Mykletun A (2010) The health status of nonparticipants in a populationbased health study the hordaland health study. Am J Epidemiol 172:1306-1314

53. Nilsen RM, Vollset SE, Gjessing HK, Skjaerven R, Melve KK, Schreuder P, Alsaker ER, Haug K, Daltveit AK, Magnus P (2009) Self-selection and bias in a large prospective pregnancy cohort in Norway. Paediatr Perinat Ep 23:597-608

54. Allison PD (2006) Fixed effects regression models in $\mathrm{SAS}^{\circledR}$. In: Inc SI (ed) Proceedings of the thirty-first annual SAS ${ }^{\circledR}$ users group international conference. SAS Institute, Cary 